\section{Latest culture techniques: cracking the secrets of bone marrow to mass-produce erythrocytes and platelets ex vivo}

\author{
Christian A. Di Buduo, ${ }^{1}$ Alicia Aguilar, ${ }^{1}$ Paolo M. Soprano, ${ }^{1}$ Alberto Bocconi, ${ }^{1,2}$ \\ Carolina P. Miguel, ${ }^{1}$ Giovanna Mantica ${ }^{1}$ and Alessandra Balduini ${ }^{1,3}$
}

${ }^{1}$ Department of Molecular Medicine, University of Pavia, Pavia, Italy; ${ }^{2}$ Department of Chemistry, Materials and Chemical Engineering G. Natta, Politecnico di Milano, Milano, Italy and ${ }^{3}$ Department of Biomedical Engineering, Tufts University, Medford, MA, USA

\section{ABSTRACT}

S ince the dawn of medicine, scientists have carefully observed, modeled and interpreted the human body to improve healthcare. At the beginning there were drawings and paintings, now there is three-dimensional modeling. Moving from two-dimensional cultures and towards complex and relevant biomaterials, tissue-engineering approaches have been developed in order to create three-dimensional functional mimics of native organs. The bone marrow represents a challenging organ to reproduce because of its structure and composition that confer it unique biochemical and mechanical features to control hematopoiesis. Reproducing the human bone marrow niche is instrumental to answer the growing demand for human erythrocytes and platelets for fundamental studies and clinical applications in transfusion medicine. In this review, we discuss the latest culture techniques and technological approaches to obtain functional platelets and erythrocytes ex vivo. This is a rapidly evolving field that will define the future of targeted therapies for thrombocytopenia and anemia, but also a long-term promise for new approaches to the understanding and cure of hematologic diseases.

\section{Introduction}

The importance of three-dimensional (3D) tissue systems has grown substantively in recent years as laboratory tools that recapitulate the physiological architecture of native human tissues. ${ }^{1}$ The bone marrow represents a challenging organ to reproduce because of its structure and complexity within the bone cavity. ${ }^{2}$ Recent research in the field aims to overcome the problems by developing different $3 \mathrm{D}$ models for the ex vivo production of blood cells. These systems can be used to understand the process of hematopoiesis in normal conditions and disease states and as an innovative way to produce blood products for clinical needs.

More than 100 million units of blood are reported to be collected worldwide every year. Nevertheless, in no country does the contribution of volunteers succeed in coping with the growing demand, making it necessary to create alternative methods for the production of blood cells. ${ }^{3}$ There is particular interest in the possibility of producing erythrocytes and platelets.

Platelets are needed more than other blood components because they are perishable. While erythrocytes can be refrigerated and used for up to 6 weeks and plasma can be frozen for as long as a year, platelets must be kept at room temperature to maintain their shape and function, which means that they have a shelf-life of only 5 days inside transfusion bags. ${ }^{4}$ Overall, blood products for transfusion are often unavailable in low-income and middle-income countries. ${ }^{5,6}$ Furthermore, even in developed countries, outside of large and medium-sized cities, hospitals can run out of platelets and erythrocytes when donation rates are down, which occurs mainly during the summer or public health emergencies, such as a pandemic. In the last few years, emerging infectious diseases have captured the attention of the transfusion medicine community and measures have been implemented to address
Ferrata Storti Foundation

\section{Correspondence: \\ CHRISTIAN A. DI BUDUO \\ christian.dibuduo@unipv.it}

\section{ALESSANDRA BALDUINI \\ alessandra.balduini@unipv.it}

Received: September 25, 2020.

Accepted: December 11, 2020.

Pre-published: January 21, 2021.

https://doi.org/10.3324/haematol.2020.262485

(C)2021 Ferrata Storti Foundation

Material published in Haematologica is covered by copyright. All rights are reserved to the Ferrata Storti Foundation. Use of published material is allowed under the following terms and conditions:

https://creativecommons.org/licenses/by-nc/4.0/legalcode. Copies of published material are allowed for personal or internal use. Sharing published material for non-commercial purposes is subject to the following conditions: https://creativecommons.org/licenses/by-nc/4.0/legalcode, sect. 3. Reproducing and sharing published material for commercial purposes is not allowed without permission in writing from the publisher. 
concerns regarding potential transmission of prions and viruses. ${ }^{7,8}$ The recent spread of Sars-CoV-2 has had a profound impact on the number of blood donations, blood component supplies, and safety. ${ }^{9,10}$ The Sars-CoV-2 has a long incubation period and causes asymptomatic infections in most people, which poses enormous challenges in the recruitment of blood donors, necessitating the implementation of new screening guidelines for hemovigilance. Indeed, during the lockdown, blood transfusion centers experienced a dramatic drop in the number of volunteers almost worldwide. Furthermore, several agents first described decades ago still represent ongoing blood safety risks that have not been adequately addressed. These include several species of Babesia known to cause human infections, which are being reported more frequently every year, especially in the USA. ${ }^{11}$

Given all this background, ex vivo manufacture of erythrocytes and platelets is becoming an increasingly attractive approach for transfusion medicine. In this review, we discuss the most recent scientific knowledge about mechanisms of erythrocyte and platelet production and technological advances in the field of bioengineering, which together have led to the development of new laboratory tools that mimic human bone marrow with different levels of complexity. The breakthrough of these approaches will lead to the generation of highly defined and controlled microenvironments in a clinical-grade condition for producing blood cell units on demand for transfusions.

\section{Looking inside the bone marrow: an overview on the origin of blood platelets and erythrocytes in vivo}

The formation of blood cells from hematopoietic stem cells (HSC) occurs within the bone marrow through a series of ever more differentiated progenitors under the tight control of soluble and environmental factors that cooperate in a framework known as the hematopoietic niche. ${ }^{12}$ Within this context, millions of platelets and erythrocytes are generated each day from a common megakaryocyte-erythroid progenitor cell that is recruited towards final differentiation by thrombopoietin or erythropoietin (Figure 1). ${ }^{13}$ A subset of long-term HSC with restricted myeloid-repopulating activity committed to the erythro-megakaryocytic lineage has also been postulated. ${ }^{14,15}$

During their process of differentiation, both cell lineages undergo characteristic morphological changes that lead to the respective lineage consolidation. These include nuclear polyploidization and cellular enlargement with the development of cytoplasmic granules and the demarcation membrane system for megakaryocytes ${ }_{16}^{16}$ while cell size reduction and chromatin condensation accompanied by increased production of hemoglobin can be observed in erythroblasts. ${ }^{17}$ Both cell lineages face one crucial final step at the end of their maturation. For megakaryocytes this is elongation of thin pseudopods, known as proplatelets, within the lumen of bone marrow sinusoids, where platelet detachment from the proplatelet shaft can be attributed mainly to turbulent blood hydrodynamics and fluid shear. ${ }^{18} \mathrm{~A}$ recent study demonstrated that membrane budding also contributes to supply the platelet biomass. ${ }^{19}$ For erythrocytes the crucial step entails expulsion of the nucleus from erythroblasts, which leads to the formation of reticulocytes, and the loss of organelles and ribosomes through autophagy/exosome-combined pathways. ${ }^{20}$

While cytokine-mediated priming is important to drive hematopoietic cell commitment, the final steps are strictly dependent on the interplay of different environmental cues, including cell-to-matrix and cell-to-cell interactions. ${ }^{21,22}$ Both platelet and erythrocyte production are profoundly influenced by the composition and stiffness of the extracellular matrix, which can model cell behavior through mechanical and chemical signals via integrins and mechano-sensitive ion channels. ${ }^{23-25}$ The protein tangle shaping the extracellular matrix is mainly composed of different types of collagen (I, II, III, V, XI), fibronectin, laminins, and glycosaminoglycans, continuously remodeled in physiology and disease through specific proteases, such as matrix metalloproteinases. ${ }^{26}$ It has been demonstrated that the softest substrates of the extracellular matrix, such as fibronectin and type IV collagen, mainly located in the medullary cavity and around sinusoids, support megakaryocyte proliferation and proplatelet formation. In contrast, the endosteal surface, which is asymmetrically enriched with the stiff type I collagen, prevents platelet production..$^{27,28}$ Regarding erythropoiesis, the interaction with fibronectin also supports cell proliferation and protects from apoptosis. ${ }^{29}$ Specialized niches localized throughout the intratrabecular space support erythrocyte maturation. Early erythroid progenitors are closely associated with perisinusoidal leptin receptor-positive stromal cells that secrete stem cell factor to support their maintenance, ${ }^{30}$ while the process of enucleation takes place within erythroblastic islands, hematopoietic sub-compartments composed of erythroblasts surrounding a central macrophage. ${ }^{31,32}$ Here, the interaction between macrophages and erythroblasts, mediated by the erythroblast-macrophage protein and integrins, is required to facilitate proliferation and differentiation and provide iron to the erythroblasts. ${ }^{33}$

In the light of all this knowledge, the top three challenges facing biomedical research today aimed at developing clinically relevant tools for ex vivo blood cell production are: (i) finding appropriate sources of stem cells; (ii) identifying efficient culture conditions for their commitment; (iii) mimicking relevant features of the bone marrow microenvironment to support the final stages of erythrocyte and platelet production.

\section{The artificial cell and stem cell pipelines for obtaining functional platelets and erythrocytes in vitro}

Artificial blood cell production and hematopoietic induction of stem cells have been studied to generate in vitro fully functional platelets and erythrocytes (Figure 2).

Synthetic platelets able to adhere to subendothelial structures have been constructed by functionalizing polymeric, liposomal or discoid albumin particles with recombinant glycoproteins or small peptides that bind to von Willebrand factor and collagen. ${ }^{34}$ More recent advances in the field include: highly deformable microgel platelet-like particles, which tenaciously bind to fibrin fibers promoting clot contraction and stability; ${ }^{35}$ platelet-like nanoparticles, whose discoidal shape and flexible exterior enhance platelet marginalization and aggregation; ${ }^{36}$ and artificial dense granules consisting of liposomes that release factors 


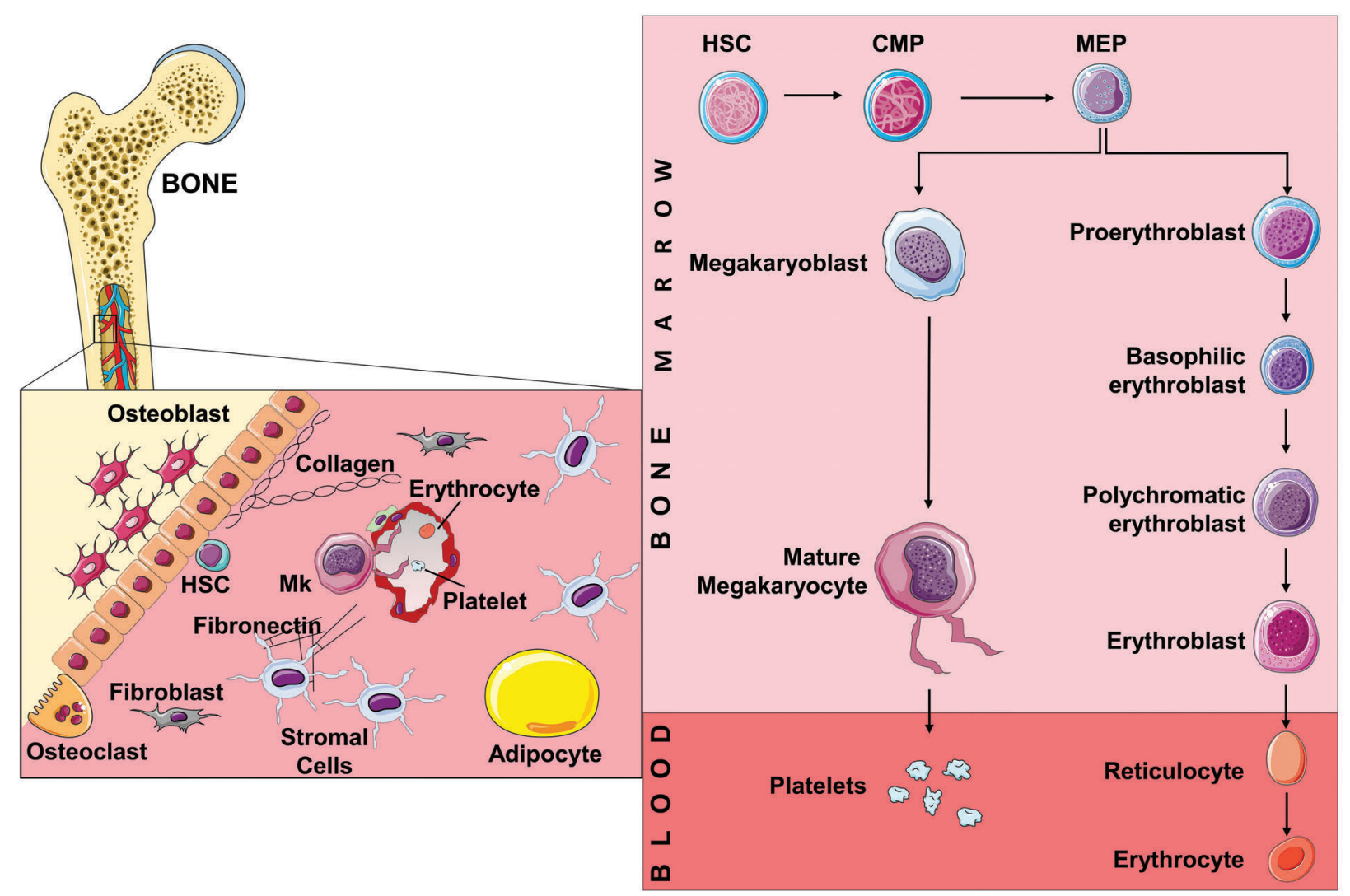

Figure 1. Bone marrow hematopoiesis. Schematic representation of the adult hematopoietic stem cell niche, showing various cell types and extracellular matrix components that influence the differentiation of blood progenitors. The hierarchical differentiation pathways of megakaryopoiesis and erythropoiesis are highlighted. Megakaryopoiesis is typically characterized by an increase in cell size and ploidy, resulting in the final extension of long pseudopods, called proplatelets, which release platelets into the bloodstream. Erythropoiesis entails several morphological and structural changes that give rise to basophilic, polychromatophilic and acidophilic erythroblasts. At the end of the terminal maturation reticulocytes are released into the bloodstream where they complete their maturation into mature erythrocytes. Mk: megakaryocyte; HSC: hematopoietic stem cell; CMP: common myeloid progenitor; MEP: megakaryocyte-erythroid progenitor. The figure was created using Servier Medical Art templates licensed under a Creative Commons Attribution 3.0 Unported License (https://smart.servier.com).

activating the coagulation cascade. ${ }^{37}$

Hemoglobin-based oxygen carriers, either human or bovine, have been proposed as erythrocyte surrogates, but none has been licensed by the Food and Drug Administration because of severe thrombotic adverse effects caused by the nitric oxide-scavenging effect of the hemoglobin molecule. ${ }^{38}$ However, successful cases of compassionate usage have been reported, ${ }^{39,40}$ and one of these products is currently used in South Africa in emergencies or when there is a clinical contraindication to blood transfusion. ${ }^{41}$

Engineered nanostructures that mimic biophysical actions of platelets and erythrocytes are therefore of interest. Advantages of their use would include no need of refrigeration and of blood grouping and matching, but they are not effective as native cells. Indeed, the functions of native cells are not easy to reproduce and thus much more effort has been focused on finding reliable sources of stem cells to be differentiated in vitro.

Animal models have been widely used. Megakaryocytes and erythrocytes can be obtained by flushing murine and rat femora or differentiated from murine fetal liver progenitors. $^{42,43}$ These are invaluable cell sources for studying the basic mechanisms of hematopoiesis and for providing proof of principle of new translational approaches for making blood cells available for transfusion. However, beyond ethical issues related to their intensive use in sci- ence, interspecies differences can render animals poor predictors of human physiology and their usage for clinical purposes is not conceivable.

Umbilical cord blood is a rapidly available source of human HSC that can be efficiently differentiated into primary cultures of erythroblasts or megakaryocytes. ${ }^{44,45}$ Umbilical cord blood HSC have been used to establish immortalized human erythroid progenitor cell lines able to produce enucleated erythrocytes. ${ }^{46}$ The main practical advantages of using umbilical cord blood are the relative ease of procurement, the lowest possibility of viral contamination and the absence of risk for mothers and donors. Nevertheless, active limitations remain the dependence on donors and the restricted availability to research teams. Finally, umbilical cord blood CD34+ cells are stem cells of fetal/neonatal origin that give rise to cells that have distinct features from those of adult cells, such as a high proliferation rate and high percentages of fetal hemoglobin in the case of erythrocytes. ${ }^{47-50}$

Methods for obtaining human adult megakaryocytes from peripheral blood or bone marrow HSC have been tested and have provided invaluable data about mechanisms of platelet production in disease states due to inherited or acquired mutations in genes relevant for the control of megakaryopoiesis. ${ }^{51}$ Giarratana et al. also used peripheral blood HSC to generate a homogeneous population of erythrocytes that were functional in terms of deformabil- 
ity, enzyme content, capacity of their hemoglobin to fix/release oxygen, and expression of blood group antigens. ${ }^{52}$ Nevertheless, these cell sources usually derive from clinical procedures and can be rare and difficult to obtain. They require a continuous supply of donors due to the limited expansion potential, making it difficult to hypothesize their usage for clinical applications. In this regard, Trakarnsanga et al. developed an alternative approach, immortalizing early bone marrow adult erythroblasts and generating a stable line functionally identical to adult donor erythrocytes. ${ }^{53}$ This cell line revealed promising properties for manufacturing red blood cell products and as a research tool for the study of erythropoiesis in health and disease. Compared to umbilical cord blood-derived immortalized human erythroid progenitor cell lines, this source demonstrated better performance and recapitulation of adult erythropoiesis. ${ }^{54}$
The need for cells that could overcome the limits related to donor dependence have prompted researchers to use human embryonic stem cells (hESC) and human induced pluripotent stem cells (hiPSC). hESC have been grown either on stromal cells or in feeder-free and serum-free cultures to produce platelet-like particles displaying functional and morphological features comparable to peripheral blood platelets, but with limited long-term self-replication of megakaryocyte progenitors and consequent yield of platelets. ${ }^{55}$ hESC have also been proposed as a source of stem cells to generate universal red blood cells, ${ }^{56}$ but with some limitations in terms of cell survival and end-stage maturation.

Good outcomes have been obtained with hiPSC, which overcome ethical concerns related to the use of cells of embryonic origin. The physiological features of hiPSCderived megakaryocytes resemble those of peripheral

\section{CELL SOURCES}

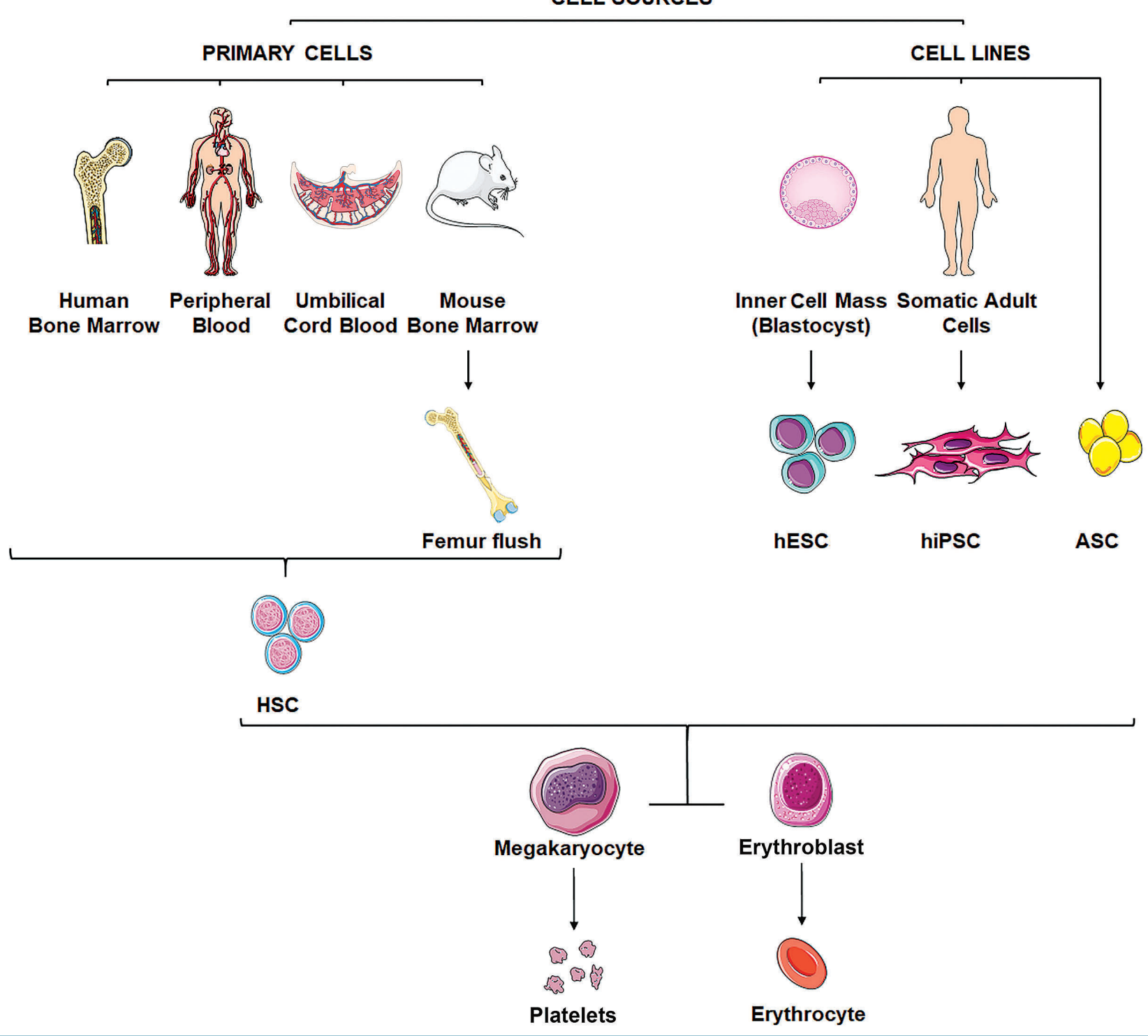

Figure 2. Overview of sources of stem cells for producing platelets and erythrocytes in vitro. Different stem cell sources have been studied for their potential to generate platelets and erythrocytes in vitro. Primary cells can be obtained either from human or mouse bone marrow, or derived from human peripheral blood and umbilical cord blood hematopoietic stem cells. Furthermore, immortalized cell lines have been generated from human embryonic stem cells, human-induced pluripotent stem cells and adipose-derived mesenchymal stromal/stem cell lines. HSC: hematopoietic stem cells; hESC: human embryonic stem cells; hiPSC: human-induced pluripotent stem cells, ASC: adipose-derived mesenchymal stromal/stem cells. The figure was created using Servier Medical Art templates licensed under a Creative Commons Attribution 3.0 Unported License (https://smart.servier.com). 
blood platelets in many aspects, including morphology, expression of lineage-specific antigens, and ability to aggregate in response to agonists. ${ }^{18,57}$ A major advantage of hiPSC is the possibility of using human leukocyte antigenmatched hiPSC or cells genetically engineered to lack certain antigens in order to produce platelets that would not be rejected by the recipient. ${ }^{58}$ Alternatively, as hiPSC can be generated from any donor, they are theoretically suitable for generating a bank of phenotypically matched erythrocytes. Various research groups have published methods for generating red blood cells from hiPSC ${ }^{59,60}$ albeit with some concerns about the expression of hemoglobin and enucleation potential, which could not be achieved in the context of in vitro differentiation, unless in the presence of feeder cells or after injection into mice recipients. Therefore, although very attractive, both hESC and hiPSC might not represent the best choice of stem cells for producing blood components. In most cases the differentiation protocols are long and expensive. Indeed, high costs for generating, validating, and maintaining these cell lines should be considered. ${ }^{61}$ Finally, there is still the concern that any cellular product derived from them could be oncogenic or teratogenic, ${ }^{62}$ given the potential genomic instability of these stem cell lines.

To overcome these limitations, the latest stem cell source to be investigated has been adipose tissue, which has the advantage of being ubiquitously available and easily accessible in large quantities with minimally invasive harvesting procedures. Ono-Uruga et al. recently demonstrated that the production of endogenous thrombopoietin is involved in megakaryocyte differentiation and platelet production from adipose-derived mesenchymal stromal/stem cell lines. ${ }^{63}$ The lack of specific markers for the identification and isolation of this subset of stem cells and the absence of standardized isolation and culture protocols make it difficult to translate this approach into different laboratories. ${ }^{64}$

\section{Finding the route towards differentiation: cytokines instruct but are not enough}

It is conceivable that a regular, non-donor-derived supply of erythrocytes and platelets could be achieved in the near future, thus making it necessary to define the best culture conditions to maximize the yield of platelets generated per single megakaryocyte, as well as the number of enucleated erythrocytes obtained in vitro (Figure 3).

Recombinant human thrombopoietin is commonly used to produce megakaryocytes. ${ }^{65-67}$ Thrombopoietin analogs, such as eltrombopag and romiplostim, have been tested in culture but their use for ex vivo platelet manufacturing is still limited because they can induce proliferation of immature progenitors. ${ }^{68,69}$ Thrombopoietin and its analogs are combined with a wide variety of cytokines including stem cell factor and interleukins (e.g., IL-3, IL-6, IL-11) which synergize to produce very pure populations of mature megakaryocytes forming proplatelets without the need for serum supplementation or co-culture with

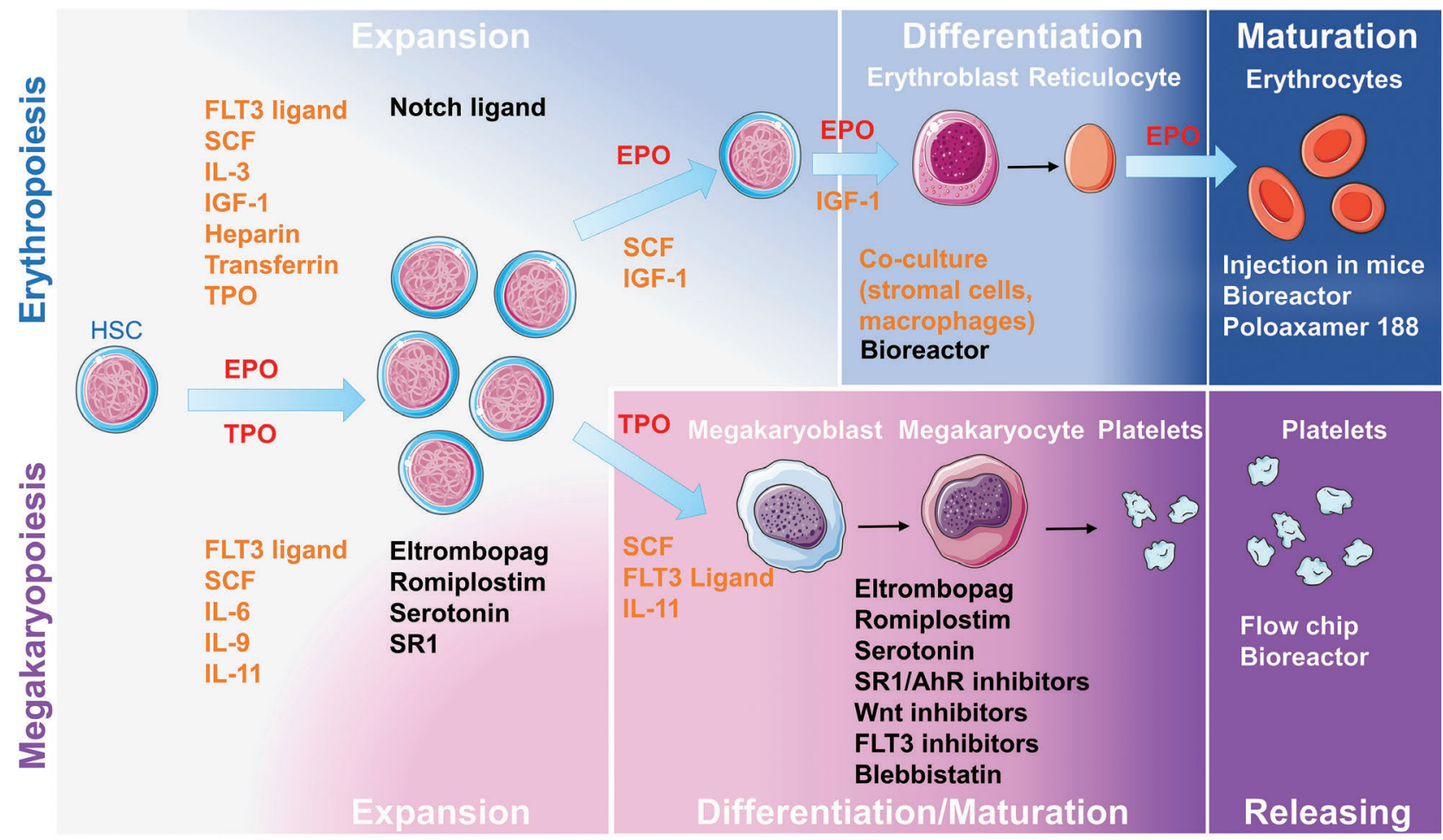

ESSENTIAL

BASIC

OTHER/NEW STRATEGY

Figure 3. Overview of soluble factors used in the production of platelets and erythrocytes in vitro. Different cocktails of cytokines, pharmacological agents and/or co-culture with feeder cells have been used to generate platelets and erythrocytes in vitro. HSC: hematopoietic stem cell; FLT3: Fms related tyrosine kinase 3; SCF: stem cell factor; IL: interleukin; IGF-1: insulin-like growth factor-1; TPO: thrombopoietin; EPO: erythropoietin; SR1: stemRegenin 1; AhR: aryl hydrocarbon receptor. The figure was created using Servier Medical Art templates licensed under a Creative Commons Attribution 3.0 Unported License (https://smart.servier.com). 
feeder cells. ${ }^{65-67}$ Other strategies include the use of serotonin, which supports megakaryocyte maturation and proplatelet formation by activating biochemical pathways and through modulation of cytoskeleton dynamics. ${ }^{70}$ The cytoskeleton is responsible for controlling membrane stiffness and resistance to deformation of megakaryocytes induced by environmental pressures. Inhibition of the actomyosin cytoskeleton by blebbistatin and Rho/ROCK inhibitors can soften the membrane and facilitate the fragmentation of megakaryocytes. ${ }^{71,72}$ However, given the importance of cytoskeleton remodeling during the whole process of differentiation, such treatments should be administrated only to mature megakaryocytes at the stage of proplatelet formation. StemRegenin1, an antagonist of the aryl hydrocarbon receptor, was shown to specifically increase the expansion of $\mathrm{CD} 34^{+} \mathrm{CD} 41^{\text {low }}$ early megakaryocytic progenitors and to promote the capacity to generate proplatelets and platelet-like elements. ${ }^{73}$ Subsequently, a high throughput screening by Seo et al. identified new inducers of megakaryocyte maturation and platelet production, such as CH223191, Wnt-C59 and TCS359, respectively inhibitors of the aryl hydrocarbon receptor and of the Wnt and Fms-like tyrosine kinase 3 pathways. ${ }^{74}$ However, studies on their mechanisms of action are needed before a conceivable application for clinical purposes.

Most of the cited compounds have been studied in liquid cultures that lack the shear forces of blood flow to support the elongation process and platelet release. To face this challenge, microfluidic chips with flow chambers and fenestrated barriers functionalized with extracellular matrix components have been proposed. ${ }^{75-77}$ Thon et al. established the first microfluidic chip. The device was made of transparent silicon and supported high-resolution live-cell microscopy and quantification of platelet production. It consisted of upper and lower microfluidic channels separated by a $2 \mu \mathrm{m}$ fenestrated barrier. Megakaryocytes were seeded in the upper channel and extended proplatelets through the slits. ${ }^{75}$ Later on, aiming to increase the yield of collected platelets, Avanzi et al. created an innovative bioreactor made of a pseudo-3D membrane, either a nanofiber membrane or a polyvinyl chloride filter, placed between two 3D-printed flow chambers. The upper side of the membrane housed megakaryocytes, and the lower compartment was a flow chamber destined to harvest platelets. ${ }^{76}$ Blin et al. designed an evolution of these chips. ${ }^{77}$ Their microfluidic device consisted of a microchannel textured with organized micropillar arrays coated with von Willebrand factor to anchor megakaryocytes while promoting platelet rolling into the flow. All these systems were able to provide a hydrodynamic shear supporting proplatelet elongation and fragmentation into platelets, but still with low efficiency in terms of numbers for clinical application because of their micro-scale nature.

Two- to four-phase cultures using combinations of erythropoietin and various growth factors, steroids and cytokines, with or without serum and/or feeder layers have been developed to reproduce complete erythropoiesis. A multistep process is needed to control the fine balance between cell expansion, differentiation and maturation. Cell expansion at the stage of stem cell progenitors has been carried out in the presence of stem cell factor, thrombopoietin and/or Fms-like tyrosine kinase 3. ${ }^{78}$ Delta 1 Notch ligand increased the proliferation rate of early progenitors but the differentiation process was delayed in this culture. ${ }^{79}$ Insulin-like growth factor 1 has been used to promote stem cell survival and to guide erythroid differentiation, and it has been validated as a promoter of nuclear condensation but not of enucleation. ${ }^{80,81}$ Erythroblast enucleation is thought to be largely dependent on signals mediated by macrophages, but mimicking erythroblastic islands is challenging and different approaches have been proposed to enhance its occurrence in vitro. The basic method consists of seeding erythroid cells with feeder cells, such as murine and human stromal cells or human monocyte-derived macrophages, but differing effects on maturation and enucleation have been reported. ${ }^{49,82,83}$ Recently, Lopez-Yrigoyen et al. established genetically programmed hiPSC-derived macrophages to develop an elegant approach of co-culture with umbilical cord bloodand hiPSC-derived erythroid cells that demonstrated efficient maturation and enucleation, ${ }^{84}$ although the presence of feeder cells could make it difficult to isolate pure, noncontaminated erythrocytes. A second approach is to inject erythroid precursors into immunodeficient mice in order for the cells to complete their maturation, but this is clearly not applicable for obtaining cells for clinical purposes..$^{52}$ In a third approach different protocols have been developed to produce enucleated erythroblasts in the absence of feeder cells, including culture with a cytoprotective polymer called poloxamer 188 which increases membrane stability during the enucleation process. ${ }^{85}$ The fourth approach involves seeding cells in agitated bioreactors systems. Timmins et al. used a commercially available device consisting of a rocking platform that guaranteed homogeneous mixing with low shear. ${ }^{86}$ In this system feeder cells were not essential for proficient expansion and terminal differentiation of erythrocytes. The same results were obtained with a bottle-turning device culture system ${ }^{87}$ and more recently with a stirred-tank bioreactor. ${ }^{88}$ Culture in suspension is an approach that can be used for large-scale production of blood cells, including megakaryocytes. Constant agitation during culture, generated by a rotary cell culture system and stirred spinner flasks, demonstrated the ability to produce platelets. ${ }^{89}$ More recently, the group of Koji Eto used big tank bioreactors to develop a scalable, controllable, turbulent flow-based system for platelet generation. ${ }^{18}$ The breakthrough of the big bioreactors was their ability to guarantee appropriate oxygenation and prevent cell conglutination while providing dynamic flows mimicking those of the bloodstream.

In most of these conditions erythrocytes and platelets look immature; in particular, erythrocytes appear macrocytic with a large amount of fetal hemoglobin and platelets appear larger with immature granules. It is known that cultured megakaryocytes of any origin produce fewer platelets per single cell than do platelets in vivo. We can hypothesize that this is because they miss the physical environment of the bone marrow which drives maturation of HSC and the final production of erythrocytes and platelets in vivo. Thus, one of the major challenges in this field of research is to implement culture protocols with systems able to provide the most favorable conditions for mimicking the bone marrow hematopoietic niche.

\section{The rise and perspectives of the three-dimensional bone marrow mimic}

In the last decades, mechano-biological studies have consolidated the importance of the physical environment, 
namely 3D structure, topography, local stiffness, and physical constraints, as a guiding cue for controlling ex vivo survival, migration, differentiation and maturation of different cell types..$^{90}$ In 2D cultures, cells are seeded in liquid medium in a monolayer in the presence of molecules and soluble factors diluted directly in the medium, but the complexity of 3D tissues is completely lost. Currently, the best approach to try to model the 3D complexity of native human tissues ex vivo is to exploit biomaterials. Indeed, 3D environments made of fibers/nanofibers, solid scaffolds, and hydrogels have demonstrated the possibility to enhance the culture area and to induce better expansion of HSC during long-term cultures. We now discuss different approaches that have been undertaken to support erythropoiesis and megakaryopoiesis ex vivo.

\section{Erythropoiesis and erythrocyte production in three-dimensional cultures}

The study of erythropoiesis in three dimensions remains marginal. Housler et al. customized a 3D compartmental hollow fiber perfused bioreactor. ${ }^{91}$ The system was composed of a network of three independent bundles of capillaries, two of which were used for countercurrent medium perfusion and the third for oxygen and carbon dioxide transport. The bioreactor enabled expansion of erythrocyte progenitors and enucleation of erythroblasts. In an effort to try to maximize enucleation and recapitulate erythroblastic islands ex vivo, Lee et al. seeded late erythroblasts derived from umbilical cord bloodderived stem cells in different macroporous scaffolds and demonstrated the impact of the pore size on cell viability. Interestingly, they found clusters of mature erythroblasts, reminiscent of erythroblastic islands in the bone marrow, which in turn increased the maturation status and enucleation rate..$^{2}$ Fauzi et al. confirmed increased cell viability and proliferation, proposing a $3 \mathrm{D}$ alginate hydrogel associated with a rotating wall vessel system cultured with murine embryonic stem cells. This system demonstrated that early exposure to stem cell factor guides the differentiation of cells toward the erythroid lineage and allows a single-step culture for the production of definitive erythrocytes in 21 days. ${ }^{93}$ Allenby et al. further demonstrated the importance of combining the 3D architecture with flow, developing a perfused $3 \mathrm{D}$ hollow fiber bioreactor. ${ }^{94}$ Four ceramic hollow fibers were encased in a 3D polyurethane porous scaffold incorporated in a perfusion system that provided normoxic and hypoxic zones as in the bone marrow environment. Specific ports in the circuit enabled medium and egressed cell sampling for extracellular metabolic, protein, and cell analysis. The wall shear rate generated inside the system was estimated to be similar to that in murine bone marrow vasculature. The bone marrow-like environment of this bioreactor enabled cells to be seeded at high density to obtain continuous erythropoiesis. In addition to large-scale production these tools are candidates as models to study normal and abnormal erythropoiesis and for drug screening. Recently, a 3D model of erythropoiesis, made with polyurethane, was proposed to study erythroid failure in myelodysplastic syndromes. ${ }^{95}$ To mimic the erythroblastic islands and enucleation process, primary bone marrow cells from healthy subjects and myelodysplastic patients were seeded in the 3D scaffold, which had a pore size and distribution close to that of bone marrow architecture. The 3D culture enabled continuous expansion and complete maturation of erythrocytes over 4 weeks. Most importantly, culture of $\mathrm{CD} 4^{+}$cells in 3D scaffolds facilitated the greatest expansion and maturation of erythroid cells, including generation of erythroblastic islands and enucleated erythrocytes.

\section{Megakaryopoiesis and platelet production in three-dimensional cultures}

As already discussed, megakaryopoiesis is critically influenced by the mechanics and biochemical composition of bone marrow. In an attempt to mimic such a structure in vitro, Currao et al. produced 3D hyaluronan hydrogels functionalized with extracellular matrix components by photo-crosslinking. ${ }^{96}$ When cultured inside such a structure, megakaryocytes demonstrated the ability to form platelets into a collagen type IV enriched environment, while this function was almost abrogated in the presence of collagen type I. The 3D culture in hydrogel also showed, for the first time, the impact of physical constraints on megakaryopoiesis and mechano-transduction pathways. In pullulan-dextran 3D hydrogel megakaryocytes were larger and had increased ploidy and expression of lineage-specific transcription factors. ${ }^{97}$ Methylcellulose hydrogels showed that viability and maturation are directly linked to stiffness of the environment. Indeed, a stiff environment led to decreased survival and growth of megakaryocyte progenitors, while in a less stiff environment, megakaryocytes were more mature in terms of ploidy and morphology of the demarcation membrane system, which closely resembled that of bone marrow megakaryocytes. After recovery and transfer into the liquid medium, proplatelet production increased two-fold, due to the activation of mechano-transduction pathways and to a different actomyosin rearrangement. ${ }^{98}$ Subsequently, Abbonante et al. demonstrated that the activation of TRPV4 (transient receptor potential cation channel subfamily $V$ member 4), a membrane mechano-sensitive cation channel, regulates mechano-transduction pathways that, in turn, control thrombopoiesis on soft substrates. ${ }^{23}$ Specifically, human megakaryocytes cultured on soft silk scaffolds $(\leq 10 \mathrm{MPa})$ showed increased activation of TRPV4, leading to calcium influx and increased platelet production as a consequence of $\beta 1$ integrin activation and internalization and of Akt phosphorylation.

By putting together all the pieces of information, scientists have been trying to develop flow bioreactors that mimic blood flow and allow better oxygenation and distribution of cytokines and nutrients during the 3D culture. The first platelet bioreactor was presented by Sullenbarger and colleagues. It was composed of a polycarbonate chamber with three disks of woven polyester or colloidal crystal hydrogel with medium flowing under and over the disks but not through them, thus limiting shear stress. ${ }^{99}$ The culture of $\mathrm{CD} 4^{+}$cells in this bioreactor allowed a long-term production of platelets and a higher number of collected platelets. Interestingly, when the oxygen concentration within the device was set at a low level (5\%), HSC expansion was increased and platelet production was decreased. Contrariwise, culture at high oxygen tension (20\%) increased the production of platelets but lowered HSC expansion. ${ }^{100,101}$ A different combination of oxygen tensions at the beginning and at the end of the culture increased both HSC expansion and the final yield of platelets. More recently, Shepherd et al. developed a flow bioreactor based on a two-layer collagen scaffold. ${ }^{102}$ The porous, structurally 
graduated scaffold was meant to support megakaryocyte function by a bone marrow-like structure. To modulate the pore size of the collagen scaffold, a two-stage freezing technique was used, which created a variety of pore sizes with larger pores in the top layer and small pores at the bottom. Based on differential pore sizes this scaffold had a sieving capacity that enhanced the purity of the platelet output. Indeed, the bioreactor was conceived as a twin chamber culture system, whereby one side of the chamber allowed seeding of the cultured hiPSC-derived megakaryocytes into the scaffold, while cross-flow-generated shear forces were able to induce platelet release. In these conditions, platelet production was improved significantly compared to that in 2D cultures. Using a different approach, involving soft gel lithography, Kotha and colleagues were able to use collagen hydrogels as a scaffold to create a microvascular network. ${ }^{103}$ Endothelial cells were seeded in the microvascular network to form endothelial vessels with a lumen, while megakaryocytes were encapsulated directly in the type I collagen hydrogel. Megakaryocytes were able to migrate to the microvascular network and to extend proplatelets. This bioengineered device provided a tool to study the vascular-megakaryocyte interface during thrombopoiesis. However, one limitation of this model was the size of the vessel (around $100 \mu \mathrm{m}$ ), which was bigger than sinusoid vessels.

The search for biomaterials that can be chemically and mechanically tailored to entrap bioactive molecules, such as growth factors and extracellular matrix components, while retaining bioavailability, has spawned research into the use of silk fibroin as scaffolds. Silk fibroin from
Bombyx mori silkworm cocoons is a strong but elastic protein that is biocompatible, having low immunogenicity and low thrombogenicity. ${ }^{104,105}$ Our group designed a silk tube functionalized with components of the extracellular matrix that support platelet production, such as fibronectin, type IV collagen and laminin, and stromalderived factor-1 $\alpha$, surrounded by a type I collagen hydrogel and Matrigel or by a silk sponge. ${ }^{105}$ The structure of the silk sponge was closer to the medullar topography and enhanced adhesion and migration of cells within the structure. ${ }^{105}$ In the vascular compartment, the presence of stromal-derived factor- $1 \alpha$ directed the migration of mature megakaryocytes towards the silk tube. Platelets collected with flow passing through the tube were functional and still alive 4 days after collection. It was also shown that co-culture with a monolayer of endothelial cells or functionalization of the silk tube with vascular endothelial growth factor and vascular cell adhesion molecule 1 increases the yield of platelets. This model could be easily adapted to study mechanisms of normal and pathological megakaryopoiesis and for drug screening. ${ }^{69,105}$

In an attempt to scale-up platelet production for transfusions, multi-porous silk sponges have been investigated. ${ }^{106}$ These sponges were cultured within new modular flow chambers with flow passing through the different pores and megakaryocytes in direct contact with the flow. This enhanced the capacity of platelet production, because of the larger volume of perfusion that allowed an increase of the concentration of cells in the sponge and because of the softer environment functionalized with extracellular matrix components that support proplatelet formation.
IN VIVO

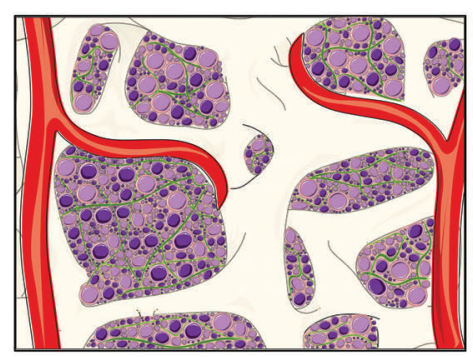

MICROFLUIDIC CHIP

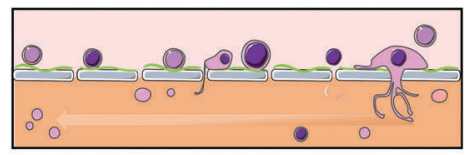

2D CULTURE

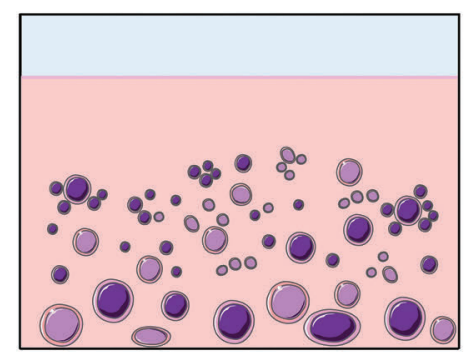

3D BIOREACTOR

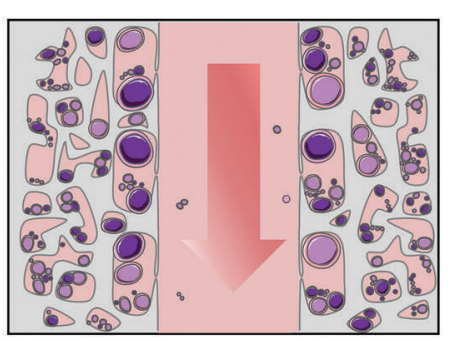

3D CULTURE

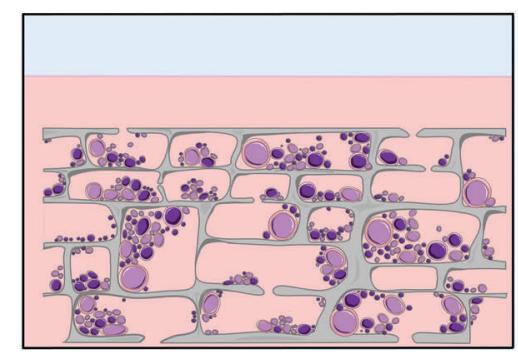

BIG TANK BIOREACTOR

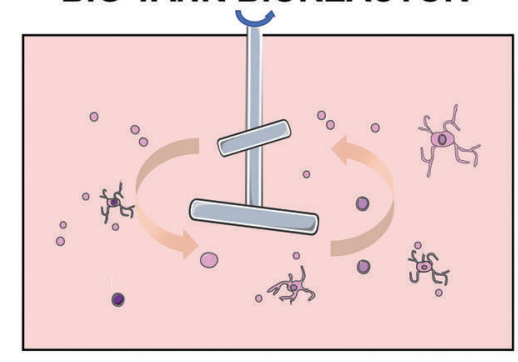

Figure 4. In vivo versus in vitro: an overview of different culture approaches for generating platelets and erythrocytes. In vivo cell maturation occurs in a complex environment in which cells experience different mechanical and biochemical cues due to cell-to-cell and cell-to matrix interactions. In the classical in vitro two-dimensional culture, cell contacts, confinement and environmental biomechanics are lost; moreover cells in contact with the plastic are artificially polarized. In the threedimensional culture, topography and stiffness can be modeled to mimic the native environment. Only cell cultured in flow conditions can recapitulate blood hydrodynamics. Microfluidic devices have the ability to enable extension of proplatelets and the release of functional platelets. Three-dimensional bioreactors combine the advantages of a three-dimensional environment with flow through the scaffold: mature cells can migrate toward the perfused compartment to release either mature erythrocytes or platelets. Cell culture in agitated or stirred-tank bioreactors has been exploited to allow large-scale production of platelets or erythrocytes. 2D: twodimensional; 3D: three-dimensional. The figure was created using Servier Medical Art templates licensed under a Creative Commons Attribution 3.0 Unported License (https://smart.servier.com). 


\section{Conclusions}

Replicating erythrocyte and platelet production ex vivo is particularly challenging in the light of the unique architecture and composition of the native bone marrow microenvironment whose biochemical and mechanical signals influence progenitor cell differentiation and function. ${ }^{12}$ Like building a house brick-by-brick, tissue-engineering approaches have combined all the knowledge collected over years of in vivo and in vitro research on hematopoiesis to develop bone marrow mimics with different levels of complexity (Figure 4). Nevertheless, there are aspects that still need to be addressed in order to increase the yield of cells produced and thereby enable clinical advances. Bone marrow mimics will need to be scaled-up several orders of magnitude to produce $3 \times 10^{11}$ platelets, ${ }^{107}$ the equivalent of one apheresis platelet unit, or $2 \times 10^{12}$ erythrocytes, the number normally contained in one unit of blood. To achieve this, the whole process has to become more costefficient to match the current prices of high-quality blood products. ${ }^{108}$ According to a current estimate, producing a number of erythrocytes in the desired range would cost thousands of dollars per unit just in consumables, without considering the investment in facilities, while the hospital costs for donated samples are in the order of magnitude of hundreds of dollars. ${ }^{109,110}$ Producing blood on a commercial scale will require substantial investment, and it will be challenging to maintain momentum in the direction of research and development of increasingly more efficient bone marrow models. Future advancement in this field will require scalable biomaterials and cell manufacturing techniques to produce blood cost-effectively in clinical-grade conditions. Costs could be reduced if specific culture components were to be produced in a bulk. The field is moving towards the discovery of novel agents, cytokines and/or chemically defined media that could be competitively priced compared to current reagents. ${ }^{74,111,112}$ However, it is clear that optimization as well as research into safety and stability are needed before clinical application. Other approaches include genetic manipulation of human pluripotent stem cells to allow enhanced cell expansion but preserved differentiation, the decrease of device manufacturing costs, the reduction of total operating volume and cytokines, and optimization of methods for recovery and concentration of the final products. ${ }^{113-115}$ However, in the short term the goal of this research is not to compete with the costs of blood donations. The most likely initial applications would be for the treatment of allo-immunized patients and those with rare blood groups. Indeed, gene therapies, with their potential to cure several benign and malignant hematologic diseases and develop cultured products to improve transfusion support for individuals with rare blood types, are currently in the therapeutic spotlight. ${ }^{116}$ Optimized 3D culture systems could provide invaluable insight for studying disease mechanisms, supporting cell growth prior to gene manipulation and testing the safety of gene-edited blood products or new pharmacological treatments on patient-derived samples before in vivo use. This is a complex and multidisciplinary task that is focused on synergistic interactions between engineering, medicine and key biological processes. Bioengineering the complex structural and dynamic microenvironment of the bone marrow is on the verge of changing the landscape of healthcare in a revolution that is only just starting.

\section{Disclosures}

No conflicts of interest to disclose.

\section{Contributions}

$A A, P M S, A B o, C P M$ and $G M$ wrote the manuscript. $C A D B$ and $A B a$ conceived the review, wrote the manuscript and gave overall direction.

\section{Funding}

This paper was supported by Cariplo Foundation (Grant 2017-0920) and by the European Commission (H2O2OFETOPEN-1-2016-2017-SilkFusion, Grant Agreement 767309). The funders had no role in the design of the review, decision to publish it, or preparation of the manuscript.

\section{References}

1. Knight E, Przyborski S. Advances in 3D cell culture technologies enabling tissue-like structures to be created in vitro. J Anat. 2015;227(6):746-756

2. Travlos GS. Normal structure, function, and histology of the bone marrow. Toxicol Pathol. 2006;34(5):548-565.

3. Dolgin E. Bioengineering: doing without donors. Nature. 2017;549(7673):S12-S15.

4. Hess JR. Conventional blood banking and blood component storage regulation: opportunities for improvement. Blood Transfus. 2010;8(Suppl 3):s9-15.

5. Custer B, Zou S, Glynn SA, et al. Addressing gaps in international blood availability and transfusion safety in low- and middleincome countries: a NHLBI workshop. Transfusion. 2018;58(5):1307-1317.

6. Allain JP. Current approaches to increase blood donations in resource-limited countries. Transfus Med. 2019;29(5):297-310.

7. Busch MP, Bloch EM, Kleinman S Prevention of transfusion-transmitted infections. Blood. 2019;133(17):1854-1864.

8. Shander A, Goobie SM, Warner MA, et al. Essential role of patient blood management in a pandemic: a call for action. Anesth Analg. 2020;131(1):74-85

9. Chang L, Yan Y, Wang L. Coronavirus disease 2019: coronaviruses and blood safety. Transfus Med Rev. 2020;34(2):75-80.

10. Stanworth SJ, New HV, Apelseth TO, et al. Effects of the COVID-19 pandemic on supply and use of blood for transfusion. Lancet Haematol. 2020;7(10):e756-e764.

11. Moritz ED, Winton CS, Tonnetti L, et al Screening for Babesia microti in the U.S blood supply. N Engl J Med. 2016;375(23): 2236-2245

12. Morrison SJ, Scadden DT. The bone marrow niche for haematopoietic stem cells. Nature. 2014;505(7483):327-334.

13. Laurenti E, Göttgens B. From haematopoietic stem cells to complex differentiation landscapes. Nature. 2018;553(7689):418-426

14. Sanjuan-Pla A, Macaulay IC, Jensen CT, et al. Platelet-biased stem cells reside at the apex of the haematopoietic stem-cell hierarchy. Nature. 2013;502(7470):232-236.

15. Yamamoto R, Morita Y, Ooehara J, et al. Clonal analysis unveils self-renewing lineage-restricted progenitors generated directly from hematopoietic stem cells. Cell. 2013;154(5):1112-1126.

16. Machlus KR, Italiano JE. The incredible jour- ney: from megakaryocyte development to platelet formation. J Cell Biol. 2013;201(6) 785-796.

17. Nandakumar SK, Ulirsch JC, Sankaran VG Advances in understanding erythropoiesis: evolving perspectives. Br J Haematol. 2016;173(2):206-218.

18. Ito $Y$, Nakamura S, Sugimoto $N$, et al. Turbulence activates platelet biogenesis to enable clinical scale ex vivo production. Cell. 2018;174(3):636-648.

19. Potts KS, Farley A, Dawson CA, et al. Membrane budding is a major mechanism of in vivo platelet biogenesis. J Exp Med. 2020;217(9):e20191206.

20. Moras M, Lefevre SD, Ostuni MA. From erythroblasts to mature red blood cells: organelle clearance in mammals. Front Physiol. 2017;8:1076.

21. Rieger MA, Hoppe PS, Smejkal BM Eitelhuber AC, Schroeder T. Hematopoietic cytokines can instruct lineage choice. Science. 2009;325(5937):217-218.

22. Yu VW, Scadden DT. Hematopoietic stem cell and its bone marrow niche. Curr Top Dev Biol. 2016;118:21-44

23. Abbonante V, Di Buduo CA, Gruppi C, et al. A new path to platelet production through matrix sensing. Haematologica. 2017;102(7): 
$1150-1160$

24. Leiva O, Leon C, Kah Ng S, Mangin P, Gachet C, Ravid K. The role of extracellular matrix stiffness in megakaryocyte and platelet development and function. Am J Hematol. 2018;93(3):430-441.

25. Ward CM, Ravid K. Matrix mechanosensation in the erythroid and megakaryocytic lineages. Cells. 2020;9(4):894.

26. Bonnans C, Chou J, Werb Z. Remodelling the extracellular matrix in development and disease. Nat Rev Mol Cell Biol. 2014;15(12):786-801.

27. Malara A, Gruppi C, Rebuzzini P, et al. Megakaryocyte-matrix interaction within bone marrow: new roles for fibronectin and factor XIII-A. Blood. 2011;117(8):2476-2483.

28. Balduini A, Pallotta I, Malara A, et al. Adhesive receptors, extracellular proteins and myosin IIA orchestrate proplatelet formation by human megakaryocytes. I Thromb Haemost. 2008;6(11):1900-1907.

29. Eshghi S, Vogelezang MG, Hynes RO, Griffith LG, Lodish HF. Alpha4beta1 integrin and erythropoietin mediate temporally distinct steps in erythropoiesis: integrins in red cell development. J Cell Biol. 2007;177(5):871-880.

30. Comazzetto S, Murphy MM, Berto S, Jeffery E, Zhao Z, Morrison SJ. Restricted hematopoietic progenitors and erythropoiesis require SCF from leptin receptor+ niche cells in the bone marrow. Cell Stem Cell. 2019;24(3):477-486.

31. Takaku T, Malide D, Chen J, Calado RT, Kajigaya S, Young NS. Hematopoiesis in 3 dimensions: human and murine bone marrow architecture visualized by confocal microscopy. Blood. 2010;116(15):e41-55.

32. Chasis JA, Mohandas N. Erythroblastic islands: niches for erythropoiesis. Blood. 2008;112(3):470-478.

33. de Back DZ, Kostova EB, van Kraaij M, van den Berg TK, van Bruggen R. Of macrophages and red blood cells; a complex love story. Front Physiol. 2014:5:9.

34. Modery-Pawlowski CL, Tian LL, Pan V, McCrae KR, Mitragotri S, Sen Gupta A. Approaches to synthetic platelet analogs. Biomaterials. 2013;34(2):526-541.

35. Brown AC, Stabenfeldt SE, Ahn B, et al. Ultrasoft microgels displaying emergent platelet-like behaviours. Nat Mater. 2014;13(12):1108-1114.

36. Anselmo AC, Modery-Pawlowski CL, Menegatti S, et al. Platelet-like nanoparticles: mimicking shape, flexibility, and surface biology of platelets to target vascular injuries. ACS Nano. 2014;8(11):1124311253.

37. Donovan AJ, Kalkowski J, Szymusiak M, et al. Artificial dense granules: a procoagulant liposomal formulation modeled after platelet polyphosphate storage pools. Biomacromolecules. 2016;17(8):2572-2581.

38. Natanson C, Kern SJ, Lurie P, Banks SM, Wolfe SM. Cell-free hemoglobin-based blood substitutes and risk of myocardial infarction and death: a meta-analysis. JAMA. 2008:299(19):2304-2312.

39. Hardy JF, Bélisle S, Van der Linden P. Combined blood substitute and erythropoietin therapy in a severely injured Jehovah's witness. N Engl J Med. 2002;347(9):696697;author reply 696-697.

40. Raff JP, Dobson CE, Tsai HM. Transfusion of polymerised human haemoglobin in a patient with severe sickle-cell anaemia. Lancet. 2002:360(9331):464-465.

41. Mer M, Hodgson E, Wallis L, et al. Hemoglobin glutamer-250 (bovine) in South
Africa: consensus usage guidelines from clinician experts who have treated patients. Transfusion. 2016;56(10):2631-2636.

42. Merico V, Zuccotti M, Carpi D, et al. The genomic and proteomic blueprint of mouse megakaryocytes derived from embryonic stem cells. J Thromb Haemost. 2012;10(5): 907-915.

43. Liu J, Zhang J, Ginzburg Y, et al. Quantitative analysis of murine terminal erythroid differentiation in vivo: novel method to study normal and disordered erythropoiesis. Blood. 2013;121(8):e43-49.

44. Jin $\mathrm{H}$, Kim HS, Kim S, Kim HO Erythropoietic potential of CD34+ hematopoietic stem cells from human cord blood and G-CSF-mobilized peripheral blood. Biomed Res Int. 2014;2014:435215.

45. Di Buduo CA, Abbonante V, Marty C, et al Defective interaction of mutant calreticulin and SOCE in megakaryocytes from patients with myeloproliferative neoplasms. Blood. 2020;135(2):133-144

46. Kurita R, Suda N, Sudo K, et al Establishment of immortalized human erythroid progenitor cell lines able to produce enucleated red blood cells. PLoS One. 2013:8(3):e59890

47. Liu ZJ, Sola-Visner M. Neonatal and adult megakaryopoiesis. Curr Opin Hematol. 2011;18(5):330-337

48. Liu ZJ, Italiano J, Ferrer-Marin F, et al. Developmental differences in megakaryocytopoiesis are associated with up-regulated TPO signaling through $\mathrm{mTOR}$ and elevated GATA-1 levels in neonatal megakaryocytes. Blood. 2011:117(15):4106-4117.

49. Giarratana MC, Kobari L, Lapillonne H, et al. Ex vivo generation of fully mature human red blood cells from hematopoietic stem cells. Nat Biotechnol. 2005;23(1):69-74.

50. Zhang X, Ma YN, Zhang JW. Human erythroid progenitors from adult bone marrow and cord blood in optimized liquid culture systems respectively maintained adult and neonatal characteristics of globin gene expression. Biol Res. 2007;40(1):41-53.

51. Tomer A. Human marrow megakaryocyte differentiation: multiparameter correlative analysis identifies von Willebrand factor as a sensitive and distinctive marker for early $(2 \mathrm{~N}$ and $4 \mathrm{~N}$ ) megakaryocytes. Blood. 2004;104(9):2722-2727

52. Giarratana MC, Rouard H, Dumont A, et al Proof of principle for transfusion of in vitrogenerated red blood cells. Blood. 2011;118(19):5071-5079.

53. Trakarnsanga K, Griffiths RE, Wilson MC, et al. An immortalized adult human erythroid line facilitates sustainable and scalable generation of functional red cells. Nat Commun. 2017;8:14750

54. Daniels DE, Downes DJ, Ferrer-Vicens I, et al. Comparing the two leading erythroid lines BEL-A and HUDEP-2. Haematologica. 2020;105(8):e389-e394.

55. Takayama N, Nishikii $\mathrm{H}$, Usui J, et al Generation of functional platelets from human embryonic stem cells in vitro via ESsacs, VEGF-promoted structures that concentrate hematopoietic progenitors. Blood. 2008;111(11):5298-5306.

56. Ma F, Ebihara Y, Umeda K, et al. Generation of functional erythrocytes from human embryonic stem cell-derived definitive hematopoiesis. Proc Natl Acad Sci U S A. 2008;105(35):13087-13092.

57. Moreau T, Evans AL, Vasquez L, et al. Largescale production of megakaryocytes from human pluripotent stem cells by chemically defined forward programming. Nat
Commun. 2016:7:11208.

58. Suzuki D, Flahou C, Yoshikawa N, et al. iPSC-derived platelets depleted of HLA class I are inert to anti-HLA class I and natural killer cell immunity. Stem Cell Reports. 2020;14(1):49-59.

59. Kobari L, Yates F, Oudrhiri N, et al. Human induced pluripotent stem cells can reach complete terminal maturation: in vivo and in vitro evidence in the erythropoietic differentiation model. Haematologica. 2012;97(12): 1795-1803.

60. Razaq MA, Taylor S, Roberts DJ, Carpenter L. A molecular roadmap of definitive erythropoiesis from human induced pluripotent stem cells. Br J Haematol. 2017;176(6):971983

61. Huang CY, Liu CL, Ting CY, et al. Human iPSC banking: barriers and opportunities. J Biomed Sci. 2019;26(1):87

62. Ben-David U, Benvenisty N. The tumorigenicity of human embryonic and induced pluripotent stem cells. Nat Rev Cancer. 2011;11(4):268-277.

63. Ono-Uruga Y, Tozawa K, Horiuchi T, et al Human adipose tissue-derived stromal cells can differentiate into megakaryocytes and platelets by secreting endogenous thrombopoietin. J Thromb Haemost. 2016;14(6): 1285-1297.

64. Baer PC. Adipose-derived mesenchymal stromal/stem cells: an update on their phenotype in vivo and in vitro. World J Stem Cells. 2014:6(3):256-265.

65. Bhatlekar S, Basak I, Edelstein LC, et al. Anti-apoptotic BCL2L2 increases megakaryocyte proplatelet formation in cultures of human cord blood. Haematologica. 2019;104(10):2075-2083.

66. Matsunaga T, Tanaka I, Kobune M, et al. Ex vivo large-scale generation of human platelets from cord blood CD34+ cells. Stem Cells. 2006;24(12):2877-2887.

67. Balduini A, Di Buduo CA, Malara A, et al Constitutively released adenosine diphosphate regulates proplatelet formation by human megakaryocytes. Haematologica. 2012;97(11):1657-1665.

68. Currao M, Balduini CL, Balduini A. High doses of romiplostim induce proliferation and reduce proplatelet formation by human megakaryocytes. PLoS One. 2013;8(1): e54723

69. Di Buduo CA, Currao M, Pecci A, Kaplan DL, Balduini CL, Balduini A. Revealing eltrombopag's promotion of human megakaryopoiesis through AKT/ERKdependent pathway activation. Haematologica. 2016;101(12):1479-1488.

70. Ye JY, Liang EY, Cheng YS, et al. Serotonin enhances megakaryopoiesis and proplatelet formation via $\mathrm{p}$-Erk1/2 and F-actin reorganization. Stem Cells. 2014;32(11):2973-2982

71. Spinler KR, Shin JW, Lambert MP, Discher DE. Myosin-II repression favors pre/proplatelets but shear activation generates platelets and fails in macrothrombocytopenia. Blood. 2015;125(3):525-533

72. Chang Y, Auradé F, Larbret F, et al Proplatelet formation is regulated by the Rho/ROCK pathway. Blood. 2007;109(10):4229-4236

73. Strassel C, Brouard N, Mallo L, et al. Aryl hydrocarbon receptor-dependent enrichment of a megakaryocytic precursor with a high potential to produce proplatelets. Blood. 2016;127(18):2231-2240.

74. Seo H, Chen SJ, Hashimoto K, et al. A B1tubulin-based megakaryocyte maturation reporter system identifies novel drugs that promote platelet production. Blood Adv. 


$$
11
$$

